\title{
Understanding Resource Needs of Persons with Dementia and Their Caregivers
}

\author{
Rosanne DiZazzo-Miller, DrOT, OTRL* \\ Fredrick D. Pociask, PhD, PT, OCS, FAAOMPT \\ Preethy Samuel, PhD, OTRL
}

Wayne State University

* Please address correspondence to Dr. Rosanne DiZazzo-Miller, Wayne State University College of Pharmacy and Health Sciences, 259 Mack Ave Suite 2212, Detroit, MI, 48201. E-mail: ar7975@wayne.edu.

\begin{abstract}
The purpose of this study was to understand the lived experiences of family caregivers of persons with dementia in accessing resources as the disease progresses, using four focus groups. Findings indicate differences in caregiver needs by stage of dementia of the care recipient. Service professionals noted family-level dynamics as possibly hindering care and voiced concern with limited mental health services. The need to educate caregivers on the scope of available services and applicable information was clear.
\end{abstract}

Keywords: caregiver, dementia, needs, resources

In the year 2012, an estimated 15.4 million family members and friends provided 17.5 billion hours of unpaid care - valued at 216 billion dollars - to those with Alzheimer's and other dementias (Alzheimer's Association, 2013a). These statistics are profound when put in perspective of the untrained and unpaid services that these families and friends provide to their loved ones. Alzheimer's disease, which accounts for $60-80 \%$ of all dementias, is projected to affect 16 million people by 2050, and is the fifth leading cause of death for those above 65 years of age (Alzheimer's Association, 2012). The term dementia is an umbrella term that is used to describe a disease or condition that affects the brain's ability to function properly (Alzheimer's Association, 2012).

Dementia is not a specific disease, but a descriptive term for a collection of symptoms that can be caused by a number of disorders that affect the brain. People with dementia have significantly impaired intellectual functioning that interferes with normal activities and relationships. They also lose their ability to solve problems and maintain emotional control, and they may experience personality changes and behavioral problems, such as agitation, delusions, and hallucinations. While memory loss is a common symptom of dementia, memory loss by itself does not mean that a person has dementia. Doctors diagnose 
dementia only if two or more brain functions, such as memory and language skills, are significantly impaired without loss of consciousness (National Institute of Health, 2012, p.1).

According to the World Health Organization (2013), dementia is one of the most significant causes of disability among seniors globally. In addition to the functional limitations experienced by those diagnosed with this progressive disorder, their family caregivers also experience a profound negative impact on their physical, emotional and economic well-being (WHO, 2013).

Due to cognitive changes it is often unsafe for persons with dementia to live alone and they are often cared for by a family member and/or a friend. Although drugs are available for the management of some dementia related symptoms, there is no drug that can arrest the progression of the disease. Members of the immediate family, such as spouses and/or adult children, often take on the responsibility of caring for a person with dementia (Alzheimer's Association, 2013a). Research suggests that caregivers often feel unprepared after a family member receives a diagnosis of dementia (Chenoweth \& Spencer, 1986; Gibson \& Anderson, 2011; Takai, et al., 2011). Additionally, family caregivers often struggle to understand the cognitive decline and associated behavioral changes of their loved one, often ascribe a negative meaning to their relative's behavior, and blame the person rather than the disease (Vernooij-Dassen, Draskovic, McCleery, \& Downs, 2011).

Having formal support services in place to educate family caregivers could improve their understanding of the expected functional decline of their care recipients, as well as provide coping mechanisms during caregiving. Clearly there is a need for family caregivers to be educated about available formal and informal resources. For example, Ducharme et al. (2011) found that more than half of caregivers they studied had a low level of knowledge of formal services that were available to support persons with dementia. Caregivers of persons with dementia had a higher level of unmet needs for formal services and faced more barriers in accessing services when compared to other caregivers of chronically ill patients (Harmell et al., 2011; Stirling et al., 2010). However, much of the research on dementia caregivers is centered on psychological well-being such as stress, depression, and anxiety (Brodaty \& Donkin, 2009; Gaugler et al., 2008; Harmell et al., 2011, Takai et al., 2011). Although there is evidence to suggest that an increase in caregiver's mastery and self-efficacy leads to better coping, which in turn has a protective effect on their well-being (Harmell et al., 2011), few studies have explored what can be done to alleviate caregiver challenges in accessing and utilizing resources that have the potential to improve quality of life for both persons with dementia and their family caregivers.

In order to effectively direct and provide caregivers resources, it is important to ascertain caregiver needs before directing limited fiscal and human resources. The most common types of interventions available to family caregivers include psycho-educational, supportive, psychotherapy or some mix of these. However, these common sources of support do not attend to some of the everyday needs of persons with dementia and their caregivers. For example, many caregivers ask questions such as, "What do you do when they don't want to take a 
shower anymore? How do I communicate with them effectively? How am I going to afford this? What is respite and where can I find it? What legal issues might arise that I should be prepared to address?" Despite the fact that there are many agencies dedicated to supporting caregivers, caregiving-related questions can be so numerous and vast that caregivers can feel that they simply don't know what they don't know. There is a gap in the literature on how family members of persons with dementia access and utilize dementia-related caregiving resources (Smale \& Dupuis, 2004; Stirling et al., 2010). Particularly, there is sparse knowledge about resource utilization by disease stage. The purpose of this study was to describe the processes by which family members of persons with dementia living in the metropolitan Detroit area access and utilize caregiving resources and services and if utilization varies by stage of dementia of the care recipient. Additionally, findings from this study can help advance understanding of the family caregiver experience, further leveraging existing resources.

\section{Participants}

\section{Method}

Twenty-three participants (15 caregivers, 3 care recipients, and 5 service professionals) enrolled in this study after the Principal Investigator (PI) received ethical approval from the Institutional Review Board at Wayne State University and the Greater Michigan Chapter of the Alzheimer's Association. The average age of caregivers who participated in this study was 62 years and the majority were female; descriptive statistics are provided in Table 1. Eight of the caregivers were adult children of persons with dementia, five were spouses, one was a sibling and one was a friend. The majority of caregivers (87\%) reported that they were the primary caregivers of a person with dementia. Care recipients who participated in this study had a mean age of 63, which was on average one year older than their caregiver counterparts. Of the five service professionals who participated in this study, all were female with different occupations including an attorney, care counselor, respite manager, education manager and program coordinator with nursing and social work backgrounds. One service professional held a certificate of gerontology and another was a dementia training and education specialist. Four early stage caregivers and three care recipients with early stage dementia participated in the first focus group. The second and third focus groups consisted of five middle stage caregivers and six late stage caregivers respectively, while the fourth focus group included five service professionals. All focus groups were conducted in cooperation with the Alzheimer's Association, Greater Michigan Chapter. 
Table 1:

Descriptive Statistics for Caregivers and Care Recipients

\begin{tabular}{|c|c|c|}
\hline \multicolumn{3}{|c|}{ Caregiver biographical data $(\mathrm{N}=15)$} \\
\hline \multirow[t]{2}{*}{ Caregiver age in years } & Mean \pm SD & $62 \pm 9.70$ \\
\hline & Range & $48-83$ \\
\hline \multirow[t]{2}{*}{ Caregiver gender } & No. (\%) Female & $13(86.67)$ \\
\hline & No. (\%) Male & $2(13.33)$ \\
\hline \multirow[t]{2}{*}{ Caregiver race } & No. (\%) White/Caucasian & $12(80.00)$ \\
\hline & $\begin{array}{l}\text { No. }(\%) \text { Black/African } \\
\text { American }\end{array}$ & $3(20.00)$ \\
\hline \multirow[t]{5}{*}{ Caregiver role } & No. (\%) Primary Caregiver & $10(66.67)$ \\
\hline & No. (\%) Shared Primary & $3(20.00)$ \\
\hline & Caregiver & $2(13.33)$ \\
\hline & No. (\%) Secondary & \\
\hline & Caregiver & \\
\hline \multirow[t]{4}{*}{ Caregiver relationship } & No. (\%) Adult Child/In-law & $8(53.33)$ \\
\hline & No. (\%) Spouse & $5(33.33)$ \\
\hline & No. (\%) Sibling & $1(6.67)$ \\
\hline & No. (\%) Friend & $1(6.67)$ \\
\hline \multicolumn{3}{|c|}{ Care recipient biographical data $(\mathrm{N}=3)$} \\
\hline \multirow{2}{*}{$\begin{array}{l}\text { Care recipient age in } \\
\text { years }\end{array}$} & Mean \pm SD & $63.3 \pm 5.51$ \\
\hline & Range & $58-69$ \\
\hline \multirow[t]{2}{*}{ Care recipient gender } & No. (\%) Female & $2(66.67)$ \\
\hline & No. (\%) Male & $1(33.33)$ \\
\hline
\end{tabular}

In this paper, the term "family caregiver" refers to informal, non-paid, community-dwelling caregivers who are either close friends or family members of a person with dementia. The term "service professional" refers to service providers including attorneys, health care professionals and dementia program managers who received professional training and were experienced in working with persons with dementia and their family members. Service professionals were invited to participate because we believed they had valuable insights through their experiences and dealings with family caregivers. Further, they were able to provide insight into caregiver preferences with service delivery and commonly sought-after resources.

\section{Recruitment Procedures}

Recruitment took place by word of mouth, direct solicitation, and informational flyers posted at local community centers by Alzheimer's Association staff and study investigators. The Alzheimer's Association also provided local media outlets with a short summary of the study. When potential participants called the PI to inquire about the research study, eligibility requirements were reviewed and care recipient stage of dementia was determined 
based on caregiver reports of the signs and symptoms in the various stages as set forth by the Alzheimer's Association (Alzheimer's Association, 2013b). The PI also classified the care recipients based on their behaviors as reported by their caregiver into early, middle and late stage dementia, and assigned them to the appropriate focus group session. Because their cognitive decline was minimal, persons with dementia in the early stage were encouraged to attend and contribute to the first focus group discussion; persons in middle and late stage were excluded from study participation.

\section{Research Design}

This study implemented phenomenological principles in the design of focus groups, to aid in the future development of a needs-assessment to capture feelings and experiences associated with access and utilization of dementiarelated caregiving resources. We collaborated with the Greater Michigan Chapter of the Alzheimer's Association to conduct community-based focus groups to better understand caregiver needs across the stages of dementia. Four separate focus groups were conducted and data were collected using guiding questions to determine what kind of information was most and least useful to caregivers, how caregivers accessed information and resources, and their experience with access in and of itself. Caregiver and care recipient focus group questions are provided in Table 2. The focus group approach was chosen because it allowed investigators to speak directly with specific groups of participants in order to learn about the lived experiences of caregivers for persons with dementia at a specific moment in time (van Manen, 1990).

\section{Table 2:}

\section{Caregiver and Care Recipient}

\section{Focus Group Questions}

1. Please talk about the educational and training resources that you have used in the past?

2. Could you please tell us how you typically find or acquire educational and training resources?

3. Could you please tell us about the most valuable educational or training resources that you have used to date?

4. Could you please tell us about the least valuable educational or training resources that you have used to date?

5. Please describe your experience(s) in obtaining educational or training resources?

6. In hindsight, is there anything you look back on that you wish you would have known in the beginning?

7. In hindsight, is there anything that you wish was available to you?

8. Is there anything that you would like to tell us that we did not ask? 
Moreover, focus groups elicit a large amount of information in a limited amount of time, which was one of the advantages of this method of data collection (Morgan, 1997). Although some might argue that the focus group method can constrain the normal discourse of conversation, as some participants may not be open and honest when others are listening, its advantages far outweigh its disadvantages (Law et al., 1998; Morgan, 1997). For example, the interaction among participants that would otherwise not occur in individual interviews results in rich layers of data being collected about the phenomenon of interest (BradburyJones, Sambrook, \& Irvine, 2009). Further, if culture and context are important to the data collection process, the natural discussion that emerges among focus group participants when sharing lived experiences is of greater quality than if they are interviewed by a researcher from a different context (Suter, 2000).

\section{Materials}

\section{Focus Group Protocol}

A focus group protocol comprised of 20 procedural checkpoints was constructed to assure for systematic and consistent protocol administration within and among all focus groups. The protocol addressed all aspects of the focus groups ranging from confirmation of attendance, greeting participants, nametags and informed consent, through administration of focus group questions, investigator debriefings held immediately following focus groups and collection and storage of focus group materials.

\section{Participant Registration Forms}

Caregiver, early stage care recipient and subject matter expert registration forms were constructed to collect participant information. The caregiver registration form included biographical data, as well as specific caregiver information such as the number of years, days per week, and hours per day spent caregiving for care recipients. The early stage care recipient registration form included basic biographical data, as well as questions pertaining to date and nature of the diagnosis. The care provider registration form included biographical data, as well as educational background, areas of specialization, areas of research and number of hours devoted to dementia-related tasks per day and week.

\section{Focus Group Questions}

The focus group for service professionals presented participants with questions about information they thought was absolutely critical for persons with dementia and their caregivers to receive. Service professionals' questions are provided in Table 3. Investigators also asked what information they thought would be most sought after by caregivers, what would be most frightening to persons with dementia and caregivers at different stages, and what suggestions they had in making resources more accessible to both caregivers and care recipients. The questions used for caregivers inquired about the educational and training resources they used in the past, how they found them and what they considered most and least valuable educationally. All care recipient/caregiver 
groups received the same questions, whereas the service professional group had parallel but different questions appropriate to their context.

Table 3:

Service Professionals

\section{Focus Group Questions}

Per early, middle and late stage caregivers:

1. What information is absolutely critical for persons with dementia and/or caregivers to receive?

2. What is most frightening or stressful to persons with dementia and caregivers at early, mid, and late stages of the disease progression?

3. What factors and information do persons with dementia and/or caregivers most commonly seek?

4. What suggestions do you have on making resources more accessible to persons with dementia and/or caregivers?

5. Are you aware of any successful systems in place that help persons with dementia and/or their caregivers deal with this diagnosis?

6. Is there anything that you would like to tell us that we did not ask?

\section{Procedure}

All focus groups took place at the local Alzheimer's Association Chapter conference room. All participants consented to participate in the study and received a $\$ 50$ retail store gift card upon study completion. An occupational therapist of 13 years, with doctoral training and previous focus group study experience led all four focus groups. Further, the focus group moderator has extensive experience with caregivers of persons with dementia, and the same comoderator was present during each focus group to ensure consistency among groups. Video cameras, one voice recorder, and study co-investigator note-taking were used at each session to ensure consistency of data collection across groups, to assure that all participants could be properly identified during transcription, and to diminish the possibility of data loss. Participants were informed that the focus group sessions would be recorded and notes would be taken during the session. Each focus group began with a briefing about the purpose of and schedule for the study, followed by brief introductions, and the opportunity to ask questions and receive answers.

The focus groups were then conducted using the guiding questions developed for the study. The focus groups were planned for approximately two hours each but on average took up to three hours, as many participants took time to support each other rather than just restrict themselves to answering the guiding questions posed by the researchers. For example, when asked about the single most valuable resource that was provided to them one caregiver responded, "This has been the most valuable thing I've done so far...getting names of people that you're (i.e., referring to other participants) dealing with... I can go back there and ask about those people... this is helpful to me just to see other people and hear them talking about what they've done so far." 
As researchers, we did not anticipate the atmosphere that our focus groups would create, in that the caregivers learned from others in the group and were able to identify with each other in an incredibly supportive manner. For this reason, our groups ran longer than anticipated, which was at the full discretion and agreement of the participants. A break was provided midway through each focus group and refreshments and light snacks were available before and during all focus groups.

\section{Data Analysis}

The data for this study were generated from verbatim transcriptions from each of the four focus groups, as well as from researcher debriefing session notes and video recordings. Each participant's initial responses were coded in response to the guiding questions. As questions were answered and participants voiced agreement or disagreement, categories were noted on an Excel spreadsheet and responses were calculated to provide preliminary, descriptive data. After the initial data set of responses was completed, data was compared to the video recordings and debriefing notes a second time to ensure accuracy.

All transcriptions were checked for accuracy. On two occasions, member checks were completed via noted preferred method of future contact, to clarify interpretation of responses. On both occasions, participants confirmed the intents and meanings presumed by the researchers.

\section{Results \\ Early Stage Care Recipients and Caregivers}

The most frequently used resource in the early stage group was the local Alzheimer's Association trainings (see Figure 1). When asked how they preferred to get information about local resources, we found that most indicated that they preferred to get such information from health care professionals through referrals. While both persons with dementia and caregivers found the demeanor of some health care professionals to be a challenge in accessing resources, some found receiving a diagnosis from their physician to be one of the most valuable resources available to them. Specifically, psychiatric professionals were found to be least helpful early in the family's journey of diagnosis and search for answers, whereas some middle stage caregivers reported seeing a psychologist for themselves as most valuable to them on a personal level. The length of time of the psychiatric testing for diagnosis was largely referred to as "a waste of time," even though the diagnosis in and of itself was viewed as helpful. Another reported challenge was that of "information overload" as they felt that too much information was presented to them at one time. 
Figure 1:

Early Stage Caregiver and Care Recipients Most Common Responses

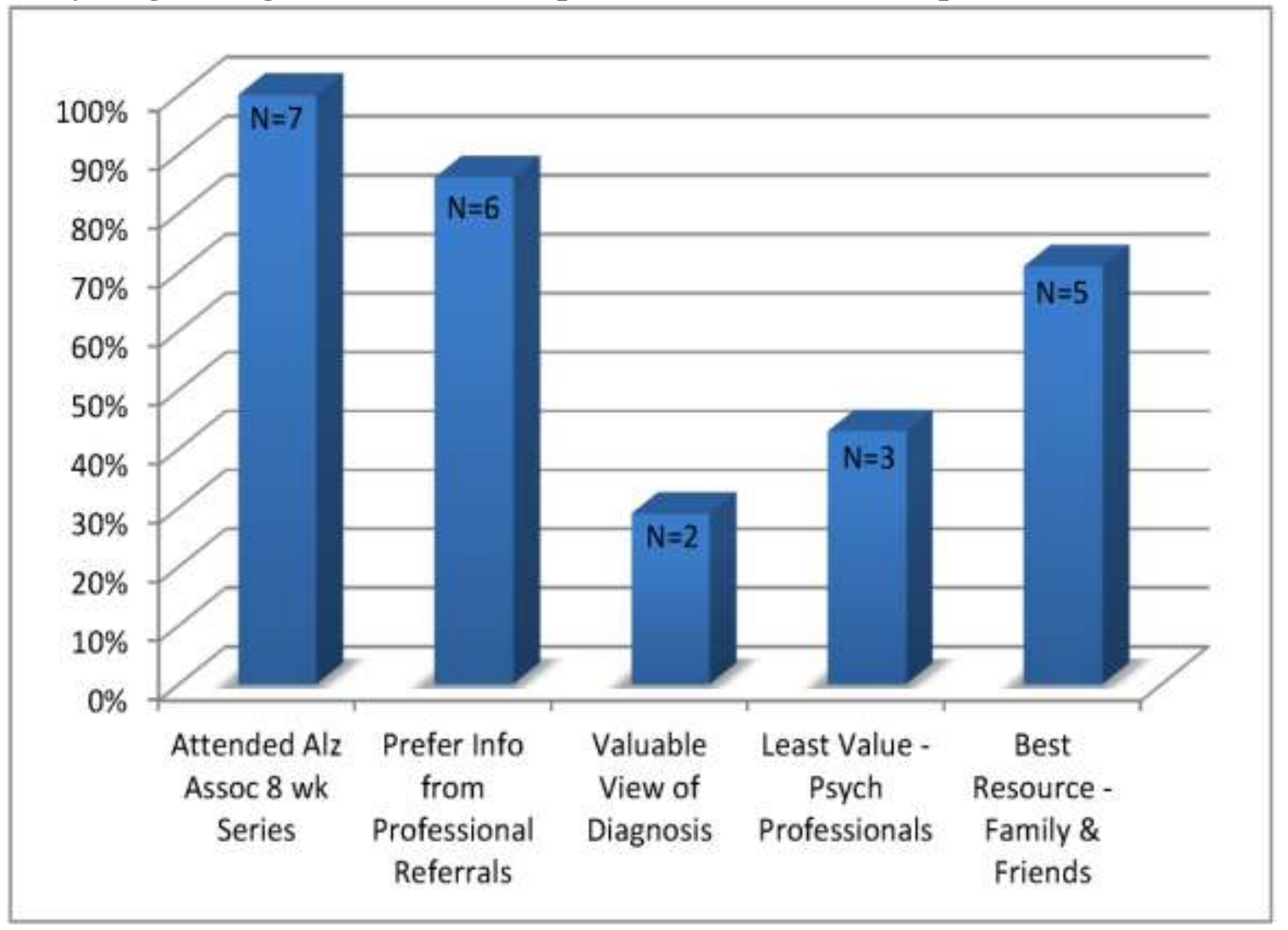

Distance and travel-related issues were identified as a barrier in accessing information during the focus group discussions. One caregiver reported they were not able to attend support groups to receive information because travel time associated with accessing the location of the support group was often unreasonable. With regards to gaining virtual support there was discussion on the challenges encountered by some in accessing informational websites that were difficult to navigate and were not perceived as user-friendly. Other barriers in accessing information included a perceived lack of information on how to assist persons with dementia with their activities of daily living (ADLs) and the process of moving to assisted living facilities. While some caregivers discussed confusion they faced with the many available treatment options in terms of identifying best practices when caring for a person with dementia, others were more concerned with basic services and asked about resources available for low-income families.

Early stage caregivers mentioned facing many different issues when seeking support and informational resources. Some caregivers of early stage dementia also felt as though too much information was presented all at once, which presented a separate and distinct challenge at this stage. Perhaps one caregiver summed it up best when she stated, 
“I feel like everybody's wanted to walk away rather than give me answers. When you have a specific problem, then you know how to address that problem, but when it's all fuzzy, you don't know....I'd like somebody to give me a diagram."

\section{Middle Stage Caregivers}

An overwhelming theme from the discussion by this group of caregivers was a desire to have a one-stop shop as they tried to access resources. They recognized the intricacies in setting up such a resource, as evidenced by the comment, "It would be kind of tricky setting it up but . . . a basic list of things, ah that ... caregivers should do and then with in that, you know, daycare, legally, socially, with your family and so forth and so on."

While there were some overlaps in resources used, preferences in access and challenges associated with that access is best understood on a case-by-case basis when analyzing the data from this focus group. In other words, there was little to no overlap in the preferences and challenges reported by participants in this focus group. The areas of conversation that did show overlap and therefore a high percentage of reports can be seen in Figure 2. Interestingly, more middle stage caregivers voiced preference and use of the Internet in searching for resources, even though personal referral was the preferred method utilized initially and throughout all stages.

Figure 2:

Middle Stage Caregivers Most Common Responses

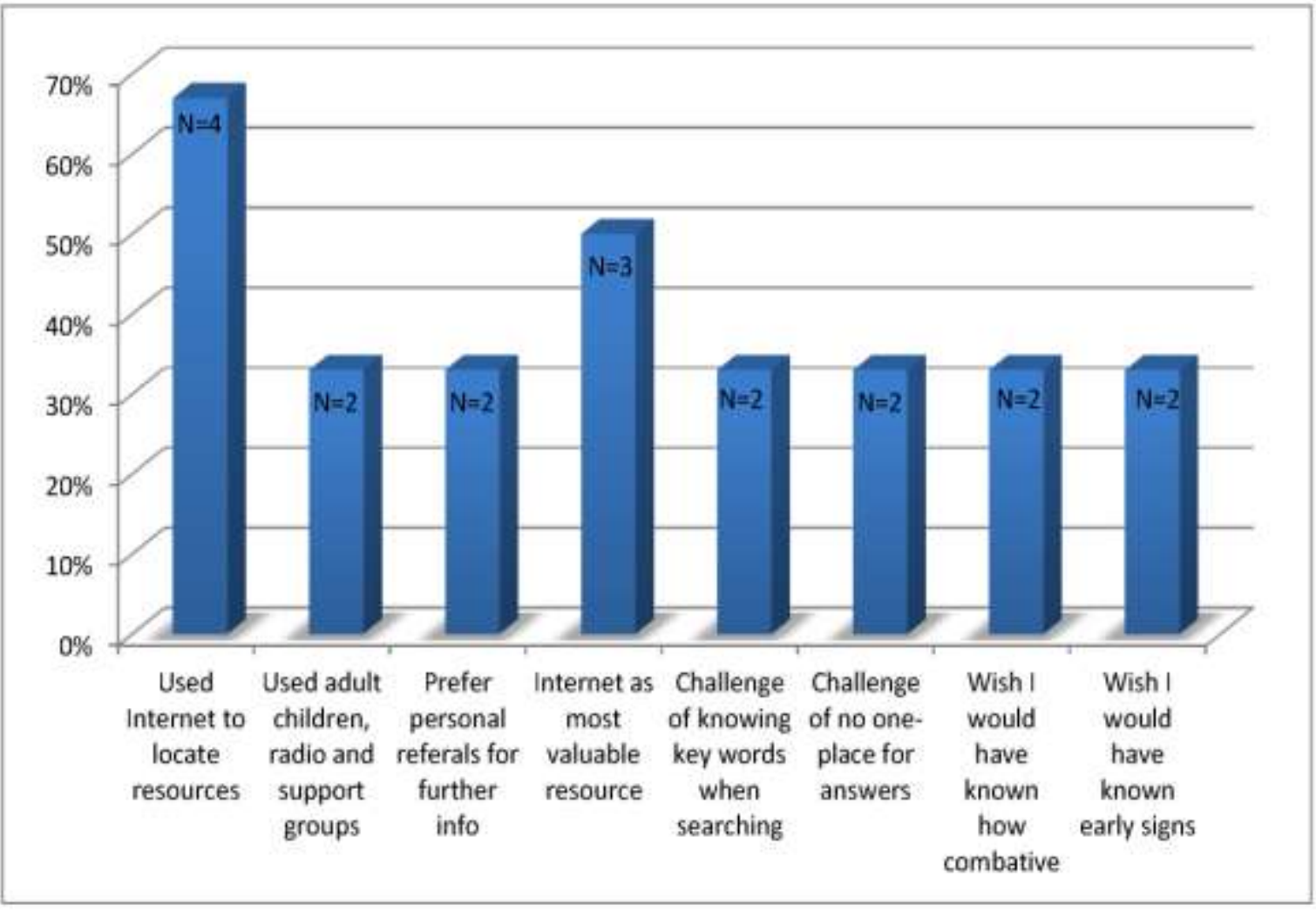


Specific resources sought after by middle stage caregivers included more locations for accessing respite care, mobility transportation resources and home health care agencies that specialized in dementia care. Although middle stage caregivers demonstrated more awareness of resources and utilized the Internet more than early stage caregivers, their continued desire to speak one-on-one with people to discuss concerns and seek out further resources was clear. The need for face-to-face support was further illustrated following a quote by a middle stage caregiver caring for his mother-in-law:

"You've got the support groups but maybe have some availability or some people that can provide some counseling, to family or to individual members because it's a lifestyle change, there's a lot of things that happened to you and, you know, in a group setting people aren't frequently as open and to have some resources and go if you need to talk to somebody....you would be able to talk to them about these issues and become aware of those things."

\section{Late Stage Caregivers}

Caregiver experiences in accessing resources differed throughout all three stages. While some groups (particularly at the middle stage of dementia) presented a wider variety of experiences, this late stage group had many similar experiences. All caregivers in this stage accessed the Internet for resources and depended mostly on family for support; viewing physicians was seen as least helpful, followed by social workers (see Figure 3). In contrast, early stage caregivers and care recipients found contact with the physician to receive diagnosis as helpful and one caregiver in the early stage group felt "blessed with a really good primary care physician [who] will give wonderful recommendations." In the later stage, family not only helped locate resources, but also provided help in coping with depression, stress, and frustration. Late stage caregivers found the most help from the Alzheimer's Association and the various programming the Association offered, including respite and day programs. These focus group participants noted both the use and importance of financial and legal planning as well.

Caregivers in the late stage voiced extreme frustration in accessing resources and clearly echoed the previous suggestion of having a single source that provides direction, advice and a list of all possible resources. One late stage caregiver described the difficulty in locating resources as an issue "probably with my own energy and denial. I guess I just need to spend more time on it. There is no one source...everything that I've uncovered has been difficult." On more than one occasion these caregivers pointed to the importance of having to learn and use "key words" (i.e., professional jargon): "The difficulty is just learning, I mean I feel like there are key words out there [that] you kinda have to tell people when you're calling different organizations." Not knowing these key words was agreed upon by more than one late stage caregiver as a clear challenge and possible obstacle in locating sought-after resources. Furthermore, a "one stop list" of sorts was echoed by many in this group as well, after one caregiver stated, "It would be nice if you could just go to one place and go through everything, a check list of 
every single thing you really should consider if you have someone with dementia in your family." This led another late stage caregiver to describe their ability to access resources as "feeling like you're on this endless loop, running into walls, etcetera, because you're running into walls at home in the first place."

\section{Figure 3:}

\section{Late Stage Caregivers Most Common Responses}

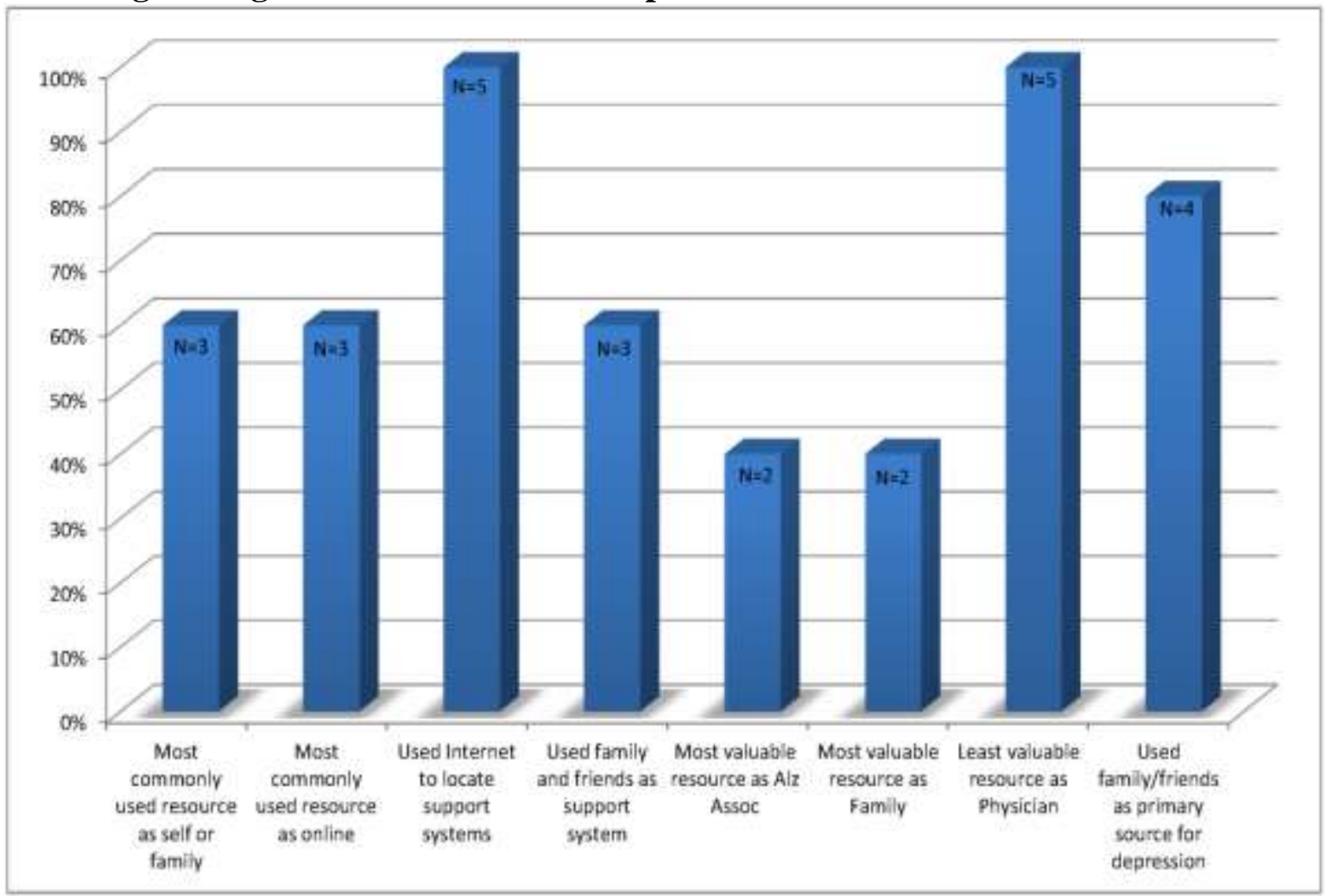

\section{Service Professionals}

All five service professionals practiced a refreshing and important use of person-first terminology in one manner or another throughout the focus group. Service professionals' responses are presented in Figure 4. Their answers echoed the earlier focus group sessions with caregivers and care recipients. Service professionals noted loss of control and loss of identity in both the caregiver and care recipient as common reports throughout all three stages, which was in line with other focus group participants' responses.

Service professionals also noted a lack of awareness of local supports. One professional said,

"There's a big disconnect that people don't know about the programs, professionals don't know. . . . People in their own communities are not aware. I think educating people locally like on some of these boards we sit on. ... There's a lot going on but I don't know if everything is totally organized well as far as pulling all these resources together, and what's out there, and those types of things." 
Lack of awareness is one of the main barriers that family caregivers face in accessing support services, similar to past studies on families of persons with developmental disabilities (Burnette, 1999; Kalyanpur \& Rao, 1991; Samuel, Hobden, LeRoy \& Lacey, 2012).

\section{Figure 4:}

\section{Service Professionals Most Common Responses}

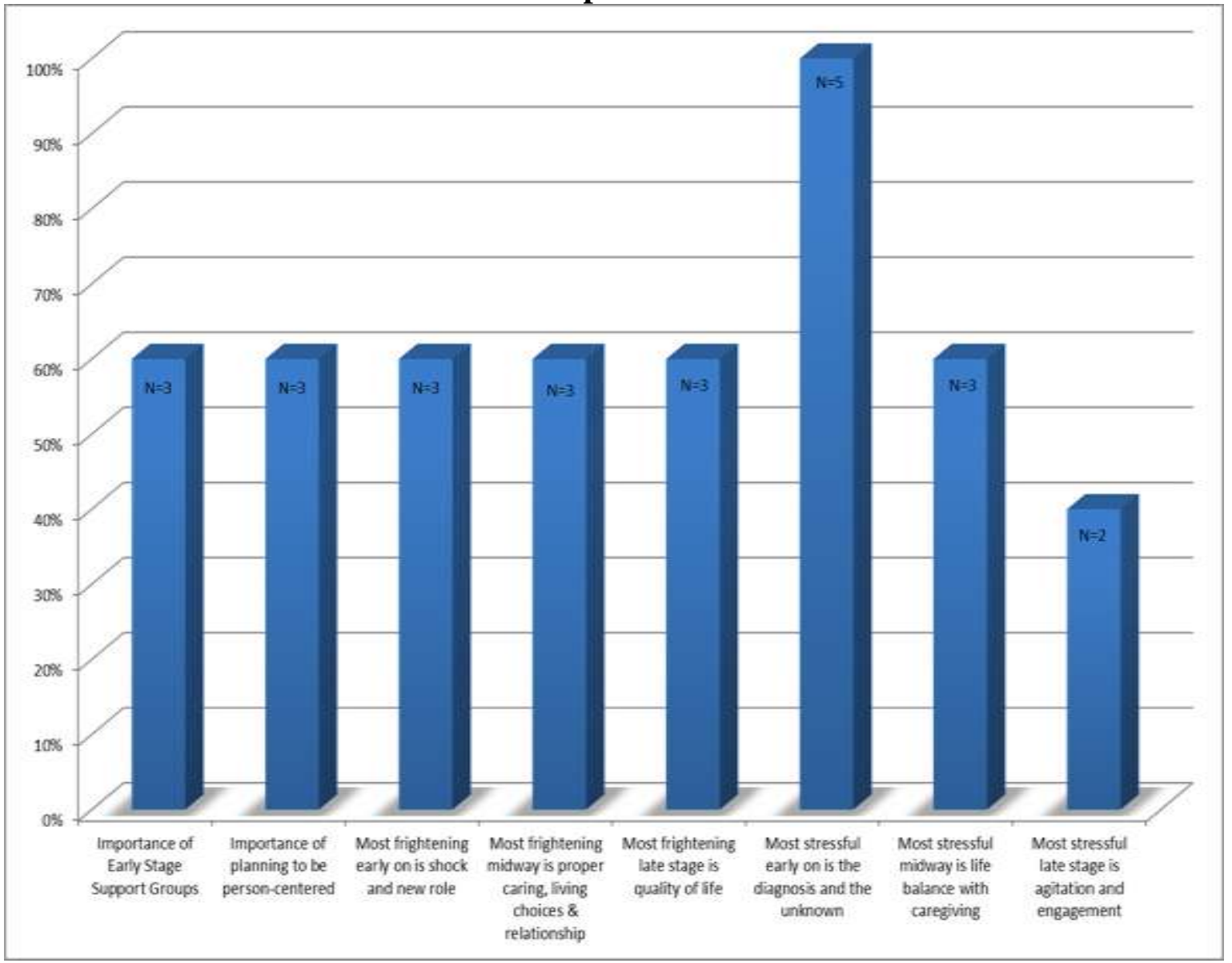

Service professionals noted the importance of all caregivers to understand "the journey [and] road ahead of them." They felt that a special focus on planning and identifying care recipient's wishes and preferences early on was crucial in accessing appropriate resources and assistance. For example one professional said, "In terms of asking for help, they (i.e., family caregivers) don't know what to ask for." This comment suggests that family caregivers will benefit from education on the scope of available support services and how these services can improve their and their care recipients' lives, before asking families to prioritize needs formally. Educating caregivers on local opportunities to get formal and informal supports as the disease progresses is an essential first step in empowering caregivers to plan effectively for the care that will be required for their care recipients with dementia. 


\section{Wishes and Desires}

At the conclusion of the focus groups we asked, "Is there anything that you would like to tell us that we did not ask?" Some of the responses were reflective of the caregiving stage.

One early stage caregiver said, "As a caregiver, how is it changing your life?" This comment shows that, in the early stage, some caregivers view the caregiving experience as transformative rather than as a burden to be shouldered. This finding is refreshing and strengthens the small body of literature that suggests that the caregiving process can be viewed as positive and meaningful (Boerner, Schulz \& Horowitz, 2004; Cohen, Colantonio \& Vernich, 2002; Smale \& Dupuis, 2004).

One middle stage caregiver said, "I've learned a lot here today . . . I I want to make sure she's (my mom) comfortable at whatever cost. I think I owe that to her. So that's why I'm glad to hear about all these things." This demonstrates that group discussions, although meant to be informative to the researchers on challenges in accessing resources faced by caregivers, had a therapeutic effect on the participants. Further, one late stage caregiver chose to answer this concluding question by asking, "How can we help?" Rather than discussing further needs and resources, an outstanding message conveyed by this group of caregivers was that they were ready to give back to their peers and professionals by sharing their life experiences, both their challenges and victories.

Service professionals were frustrated by the paucity of locally available services, particularly a need for good quality mental health services for persons with dementia, as captured by these comments: "There's nowhere for them (persons with dementia and psychiatric co-morbidities) to go." Another stated, "There's nowhere to send them (care recipients), or they're (health care professionals) not trained. Or they're just, you know, being chemically restrained. And now a lot of psych type places are not accepting people with dementia." Finally, one service professional mentioned, "It's not that there's not programs out there but it all comes back to funding."

Service professionals also described feeling overwhelmed when dealing with family level issues as suggested by the following, "The most difficult clients I see are ones that come in as families, because if it's a spouse caring for their spouse, they're having problems with their children not stepping up and helping (alternatively there is also fighting among children caring for parents). It's the fighting... that is preventing I think proper care for the person with memory loss." Another stated, "It gets ugly." A final professional suggested, "More resources are needed to look at how to deal with family dynamics and how much added stress and anxiety it causes." The need to train service professionals in familycentered care was clear.

\section{Role of Medical Professionals}

\section{Discussion}

Our finding that attitudes of medical professionals frustrate early stage family caregivers is not novel, but it further supports anecdotal evidence and converges with past studies on this topic. For example, the most noted and 
concerning issue in this study was the lack of referrals from physicians following diagnosis. This might be because medical professionals themselves may not be aware of local organizations and resources that can be helpful to families of persons with dementia. For example, a recent Canadian study found that only $13.1 \%$ of family caregivers were referred for support and counseling by their physicians (Yaffe, Orzeck \& Barylak, 2008). This responsibility should not fall on the shoulders of physicians alone; it is the responsibility of all members of the health care team. This lack of knowledge of local resources and decreased referrals is not only a problem in the dementia medical community but is also noted among other groups of service professionals, including caseworkers of families of children with disabilities (LeRoy, Johnson, \& Israel, 2004).

Furthermore, applicability of information to the family is another prominent challenge. To put this in perspective, one caregiver in the early stage focus group reported, "You go to the professionals and they give you the information but they can't do anything for you. So that's as far as that goes."

\section{Informal Supports}

Throughout all stages of dementia caregiving there was a noted reliance upon family and friends to not only provide support but resources for caregivers. This finding converges with reports from Smale \& Dupuis (2004) who found that more than half of all caregivers $(55 \%)$ received emotional support from other family members and almost as many (46\%) received this support from friends and neighbors. They also reported that about $53 \%$ of other family members practically helped the caregivers with many of the caregiving tasks for the person with dementia, and that overall, caregivers used far more informal supports than formal supports.

\section{The Internet}

Activities of daily living, transition to assisted living, resources for lowincome and evidence-based practice were noted as desired resources for caregivers in the early stage, and areas of searches for those who used the computer and Internet. Despite the numerous resources that appear after an initial Internet search for "dementia caregiver resources," many participants described either being uncomfortable and/or inexperienced with computer use, or inundated with information overload. Of equal concern, participants were often uncertain about the legitimacy and accuracy of information they were retrieving via the Internet. In our study, Internet use rose markedly from $14 \%$ in early stage, to $67 \%$ in middle stage, ending at $100 \%$ in late stage caregiving. Many studies have tested the efficacy of Internet-based support programs, as it is a reliable, cost-efficient way to provide supportive resources (Beauchamp, Irvine, Seeley \& Johnson, 2005).

The provision of reliable, accessible, cost-efficient resources will continue to be a challenge within our health care system. However, with further study and exploration, we can identify ways to aid persons with dementia and their caregivers throughout all stages of disease progression. This study is the first step in identifying resource needs for persons with dementia and their caregivers. Our 
findings may help fill in gaps of educational and training needs for this population.

\section{Limitations and Future Research}

It would have been beneficial to conduct one-on-one interviews with persons with early-stage dementia and compare their concerns with those of their caregivers instead of combining them into the same group. We recommend individual interviews with persons with dementia, as they may experience cognitive and behavioral issues not conducive to a group setting. However, within the scope of this study the use of focus groups was beneficial as the discussions among the participants resulted in rich layers of data that may not have occurred in individual interviews as highlighted by Bradbury-Jones, Sambrook, \& Irvine (2009). Another limitation to this study was our inability to use a standardized measure of evaluating the stage of dementia of the care recipient. Finally, although the purpose of this study was to explore accessing resources for caregivers in the metropolitan Detroit area, these findings may not be reflective of all caregivers in this area as the majority of participants in our study were already connected to the local chapter of the Alzheimer's Association. The needs of families who are not so well connected could be very different from what we observed.

Future research will utilize insight gained from this preliminary study to develop a survey that is expected to better capture the needs of caregivers living in the metropolitan Detroit area (i.e., as compared to a survey constructed without preliminary analysis). The planned caregiver survey will seek to recruit a large and representative number of participants in order to more readily generalize survey findings to caregivers living in the metropolitan Detroit area, and possibly to other like regions of the country.

\section{Conclusions}

As healthcare professionals, temperament, tone, and delivery are more important than anyone can possibly stress. The importance of providing a perfect balance of applicable information on disease progression is always important, but what is most valuable to persons with dementia and their caregivers is information on how this disease progression directly affects their family, daily interests and daily routines. Caregivers prefer personal, professional referrals to resources that contain the perfect balance of information and applicability without information overload. Clearly there is an undeniable need for user-friendly resources at a local and national level that can quickly and effectively connect caregivers with existing and contextually relevant educational training materials and/or care professionals. Equally as important, health care professionals need to be aware of these resources so that they can better equip family caregivers to face the many challenges encountered in their caregiving journey.

The obstacles and challenges faced by caregivers in this study in locating resources included a lack in knowledge of professional jargon in general and specific to communications needed for different agencies, and inability to muster enough energy and courage to pursue resources in the absence of a one-stop-shop 
that could provide information in a personable, one-on-one format. "Endless loops" was the term used by one caregiver to illustrate this sense of frustration in finding resources. The issue was not that resources were not developed or available but that they didn't know what they didn't know- much less where and how to find it. A diagnosis of dementia presents an end to a previous normalcy, an end to previous memories and life as everyone in the family once knew it. Dementia on its own, as one caregiver stated, is "life-changing." The continual and projected growth rate of dementia is astounding, coupled with the fact that $80 \%$ of care within the community setting is provided by informal, family caregivers (Alzheimer's Association, 2013a). A study conducted by MetLife (2006) has already indicated that it is critical to identify the needs of family caregivers and provide supports in order to allow them to continue to care for their loved ones while finding the time to care for themselves. The global cognitive, physical, emotional and functional loss experienced by persons with dementia with disease progression has a profound impact on family, especially on caregivers. Findings from this study illustrates the support desires and needs of caregivers who are simultaneously negotiating other multiple life roles- such as work and parenting- and can provide insight into answering caregivers' calls for help in closing these seemingly "endless loops".

\section{Acknowledgements}

We would like to thank the Eugene Applebaum College of Pharmacy and Health Sciences Faculty Research Award Program, the Alzheimer's Association Greater Michigan Chapter for hosting our focus groups; specifically Jean Barnas for her invaluable assistance in coordinating the focus groups, and our research assistants T. Ahmed, A. Cole, S. Czech, C. Skonieski, M. Varon, M. Moore, A. Thomas, S. Ullenbruch, L. Monterosso, A. Watkins, J. Kudla, S. Barc and T. Kimball. Thank you to Dr. Melissa Tovin at Nova Southeastern University's PhD program for her wisdom and guidance in qualitative methodology.

\section{References}

Alzheimer's Association. (2012). Alzheimer's disease facts and figures. Alzheimer's and Dementia: The Journal of the Alzheimer's Association, 8, 131-168.

Alzheimer's Association. (2013a). Alzheimer's disease facts and figures. Alzheimer's and Dementia: The Journal of the Alzheimer's Association, 9(2), 1-71. Retrieved from http://www.alz.org/downloads/facts_figures_2013.pdf

Alzheimer's Association. (2013b). Seven stages of Alzheimer's. Retrieved from http://www.alz.org/alzheimers_disease_stages_of_alzheimers.asp

Beauchamp, N., Irvine, A. B., Seeley, J., \& Johnson, B. (2005). Worksite-based Internet multimedia program for family caregivers of persons with dementia. The Gerontologist, 45(6), 793-801. 
Boerner, K., Schulz, R., \& Horowitz, A. (2004). Positive aspects of caregiving and adaptation to bereavement. Psychology and Aging, 19(4), 668-675.

Bradbury-Jones, C., Sambrook, S., \& Irvine, F. (2009). The phenomenological focus group: An oxymoron? Journal of Advanced Nursing, 65(3), 663671.

Brodaty, H., \& Donkin, M. (2009). Family caregivers of people with dementia. Dialogues of Clinical Neuroscience, 11(2), 217-228.

Burnette, D. (1999). Custodial grandparents in Latino families: Patterns of service use and predictors of unmet needs. Social Work, 44(1): 22-34.

Chenoweth, B., \& Spencer, B. (1986). Dementia: The experience of family caregivers. The Gerontologist, 26(3), 267-272.

Cohen, C. A., Colantonio, A., \& Vernich, L. (2002). Positive aspects of caregiving: Rounding out the caregiver experience. International Journal of Geriatric Psychiatry, 17(2), 184-188.

Ducharme, F., Le' Vesque, L., Lachance, L., Kergoat, M. J., \& Coulombe, R. (2011). Challenges associated with transition to caregiver role following diagnostic disclosure of Alzheimer disease: A descriptive study. International Journal of Nursing Studies, 48, 1109-1119.

Gaugler, J. E., Roth, D. L., Haley, W. E., \& Mittleman, M. S. (2008). Can counseling and support reduce burden and depressive symptoms in caregivers of people with Alzheimer's disease during the transition to institutionalization? Results from the New York University caregiver intervention study. Journal of the American Geriatric Society, 56, 421428.

Gibson, A. K., \& Anderson, K. A. (2011). Difficult diagnoses: Family caregivers' experiences during and following the diagnostic process for dementia. American Journal of Alzheimer's Disease and Other Dementias, 26(3), 212-217.

Harmell, A. L., Chattillion, E. A., Roepke, S. K., \& Mausbach, B. T. (2011). A review of the psychobiology of dementia caregiving: A focus on resilience factors. Current Psychiatry Reports, 13(3), 219-224. Retrieved from http://www.ncbi.nlm.nih.gov/pmc/articles/PMC3182821/\#_ffn_sectitle

Law, M., Stewart, D., Letts, L., Pollock, N., Bosch, J., \& Westmoreland, M. (1998). Guidelines for critical review of qualitative studies. McMaster University. Retrieved from http://wwwfhs.mcmaster.ca/rehab/ebp/pdf/qualguidelines.pdf 
LeRoy B., Johnson A. \& Israel N. (2004). The perceptions of welfare reform by Michigan families whose children have disabilities and welfare caseworkers. Social Policy Journal 3, 23-37.

Kalyanpur, M., \& Rao, S. S. (1991). Empowering low- income black families of handicapped children. The American Journal of Orthopsychiatry, 61, 523532.

MetLife. (2006). The MetLife study of Alzheimer's Disease: The caregiving experience. MetLife Mature Market Institute in conjunction with LifePlans, Inc. Retrieved from https://www.metlife.com/assets/cao/mmi/publications/studies/mmialzheimers-disease-caregiving-experience-study.pdf

Morgan, D. L. (1997). Focus groups as qualitative research. $\left(2^{\text {nd }}\right.$ ed.). Newbury Park, CA: Sage Publications Inc.

National Institute of Health. (2012, May 16). NINDS dementia information page. Retrieved from http://www.ninds.nih.gov/disorders/dementias/dementia.htm

Samuel, P. S., Hobden, K. L., LeRoy, B. W., Lacey, K. K. (2012). Analyzing family service needs to typically underserved families in the USA. Journal of Intellectual Disability Research, 56, 111-128.

Smale, B., \& Dupuis, S. L. (2004). In their own voices: A profile of dementia caregivers in Ontario. Stage 1: Survey results. Murray Alzheimer Research and Education Program (MAREP), 1-122. Retrieved from https://uwaterloo.ca/murray-alzheimer-research-and-educationprogram/sites/ca.murray-alzheimer-research-and-educationprogram/files/uploads/files/InTheirOwnVoices1-SurveyResults.pdf

Stirling, C., Andrews, S., Croft, T., Vickers, J., Turner, P., \& Robinson, A. (2010). Measuring dementia carers' unmet need for services: An exploratory mixed methods study. BMC Health Services Research, 10, 110.

Suter, E. A. (2000). Focus groups in ethnography of communication: Expanding topics of inquiry beyond participant observation. The Qualitative Report, $5(1 \& 2)$. Retrieved from http://www.nova.edu/ssss/QR/QR5-1/suter.html

Takai, M., Takahashi, M., Iwamitsu, Y., Oishi, S., \& Miyaoka, H. (2011). Subjective experiences of family caregivers of patients with dementia as predictive factors of quality of life. Psychogeriatrics, 11, 98-104. 
Van Manen, M. (1990). Researching lived experience: Human science for an action sensitive pedagogy. London, Ontario: Althouse.

Vernooij-Dassen M, Draskovic I, McCleery J, Downs M. (2011). Cognitive reframing for carers of people with dementia. Cochrane Database of Systematic Reviews, 11. Art. No.: CD005318. DOI:

10.1002/14651858.CD005318.pub2.

World Health Organization (WHO). (2013). Media centre: Dementia fact sheet. Retrieved from http://www.who.int/mediacentre/factsheets/fs362/en/.

Yaffe, M.J., P. Orzeck, and L. Barylak. (2008). Family physicians' perspectives on care of dementia patients and family caregivers. Canadian Family Physician Medecin de Famille Canadien, 54(7), 1008-15. 